\title{
Correction of beam-steering effects in low-velocity superconducting quarter-wave cavities
}

\author{
P. N. Ostroumov and K. W. Shepard \\ Physics Division, Argonne National Laboratory, 9700 S. Cass Avenue, Argonne, Illinois 60439
}

(Received 31 August 2001; published 15 November 2001)

\begin{abstract}
Superconducting cavities presently used for acceleration of ions in velocity range $\sim 0.01 c$ to $0.3 c$ (where $c$ is the speed of light) are based on quarter-wave resonators. Currently there are several design proposals in nuclear physics laboratories for application of this type of cavity for acceleration of light and heavy ions. The operating frequencies of the cavities range from $\sim 50$ to $360 \mathrm{MHz}$ to satisfy various specifications. Electrodynamics studies of the field distributions in the beam-cavity interaction area indicate appreciable dipole components of both electric and magnetic fields, especially for higher-frequency cavities. The dipole fields induce beam steering, which is a strong function of rf phase and which couples the longitudinal and transverse motion. This can result in growth in the transverse emittance of the beam. In this paper, we propose two possible methods for the correction of such dynamic beam-steering effects in quarter-wave resonators. We analyze and discuss the correction methods for the particular examples of two quarter-wave resonators operating at 57.5 and $115 \mathrm{MHz}$ designed for the driver linac of the Rare Isotope Accelerator facility.
\end{abstract}

DOI: $10.1103 /$ PhysRevSTAB.4.110101

PACS numbers: 29.17.+w, 29.27.-a, 41.75.-i

\section{INTRODUCTION}

The driver linac of the Rare Isotope Accelerator (RIA) facility [1] will use more than 100 superconducting (SC) quarter-wave resonators (QWR) operating at 57.5 and $115 \mathrm{MHz}$. A section of a typical resonator is shown in Fig. 1. This class of resonant cavity is effective for acceleration of any ion species in velocity range from $\sim 0.01 c$ to $0.3 c$, where $c$ is the speed of light. The quarter-wave structures differ from room temperature accelerating structures typically used in this velocity range in that the electric and magnetic field distributions in the accelerating gaps of the QWR are not axially symmetric. There are dipole components of both the electric and the magnetic field which can steer the beam.

We have numerically modeled the electrodynamic properties and field distributions using Microwave Studio (MWS) [2]. MWS running on modern PCs can calculate all six components of electromagnetic field distribution within the beam-cavity interaction area with a mesh size of 1-2 mm. The distributions of $E_{z}, E_{y}$, and $H_{x}$ on the axis of the $115 \mathrm{MHz}$ QWR are shown in Fig. 2. As can be seen, there is an appreciable magnetic field, $\sim 60 \mathrm{Gs}$, in the accelerating gaps.

As was pointed out in Ref. [3], in addition to the vertical electric-field steering, the $H_{x}$ component of the magnetic field can also produce substantial deflection in the vertical plane, especially for higher-frequency QWRs. Such steering effects will clearly be most pronounced for light ions.

While simple steering, or displacement of the beam centroid, can be compensated by using standard, static corrective steering elements, the present case is complicated by the phase dependence of both the electric and the magnetic fields. In a two-gap QWR the phase dependence of steering for both the electric dipole field and the magnetic field is shifted $90^{\circ}$ from the phase profile of the accel- erating process. This means that at typical operating $\mathrm{rf}$ phase angles, the steering is a strong function of rf phase and couples the longitudinal and transverse motion. As a result, the steering fields can, depending on the longitudinal emittance, induce appreciable transverse emittance growth. Such emittance growth cannot be compensated by static fields and can be a particularly serious problem in applications for high-intensity light-ion beams.

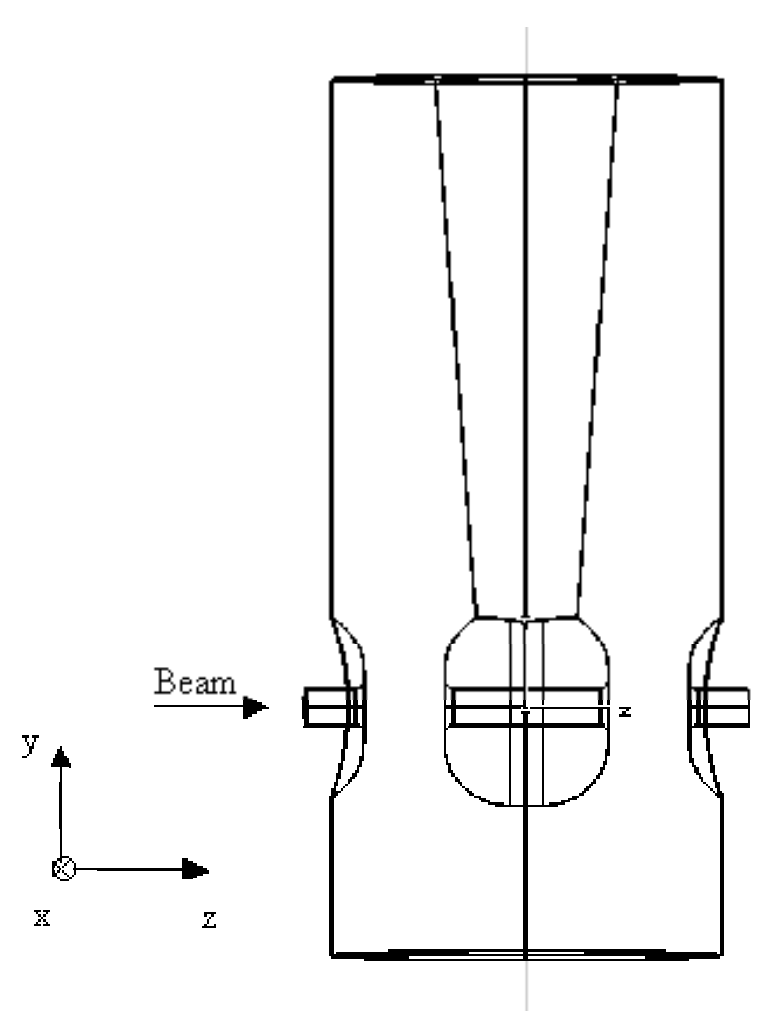

FIG. 1. $115 \mathrm{MHz}$ quarter-wave resonator, $\beta_{G}=0.15$. 


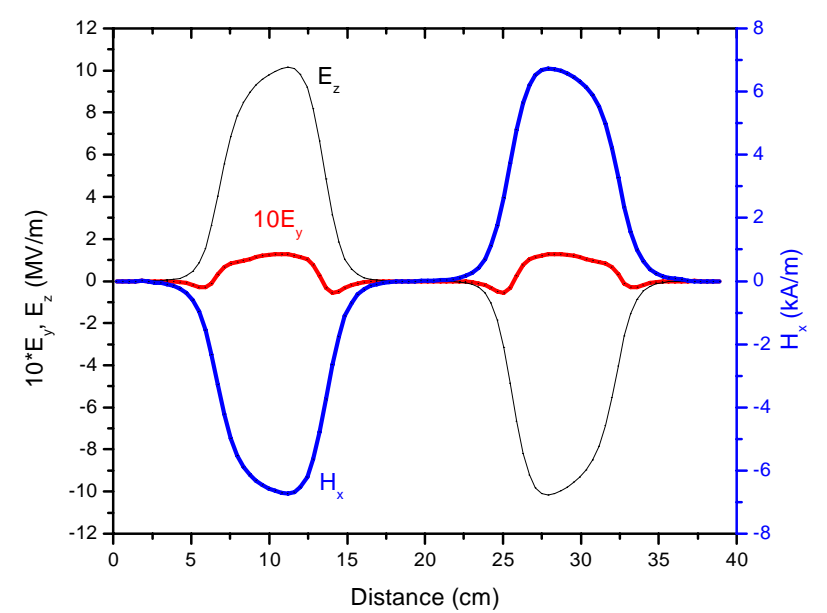

FIG. 2. (Color) Field distribution along the axis of the $115 \mathrm{MHz}$ QWR. For clarity, the magnetic field is shown with opposite sign with respect to the electric field. The amplitude of $E_{y}$ is multiplied by a factor of 10 .

In the following, we discuss the effect of dipole components of the electromagnetic field in SC cavities and pro- pose two possible methods for both correcting steering and substantially reducing emittance growth.

\section{ION MOTION IN QWR}

The equations of motion must be solved in the presence of all three components of electric and magnetic fields:

$$
\frac{d \vec{p}}{d t}=\frac{q e}{A}(\vec{E}+\vec{\nu} \times \vec{B}),
$$

where $q$ is the ion charge state, $A$ is the mass number, $e$ is the elementary charge, and $\vec{\nu}$ is the particle velocity. The code TRACK has been written in order to integrate charged particle motion in the presence of all components of the electromagnetic field except the $H_{z}$ component which is negligible in current applications. The TRACK code simulates multiparticle motion in six-dimensional phase space on the base of an iterative solution of the equation of motion as it is done in the LANA code [4,5] for the particle simulation in $\mathrm{rf}$ electric fields. The equations of motion (1) can be presented in the form

$$
\begin{aligned}
\frac{\beta \gamma}{(\beta \gamma)_{0}} & =\xi \int_{0}^{\psi} E_{z}(x, y, z) \cos \left(\varphi+\varphi_{0}\right) d \varphi, \\
x^{\prime} \frac{\beta \gamma}{(\beta \gamma)_{0}}-x^{\prime} & =\xi \int_{0}^{\psi} E_{x}(x, y, z) \cos \left(\varphi+\varphi_{0}\right) d \varphi-\chi \int_{0}^{\psi} H_{y}(x, y, z) \sin \left(\varphi+\varphi_{0}\right) d \varphi, \\
y^{\prime} \frac{\beta \gamma}{(\beta \gamma)_{0}}-y^{\prime} & =\xi \int_{0}^{\psi} E_{y}(x, y, z) \cos \left(\varphi+\varphi_{0}\right) d \varphi+\chi \int_{0}^{\psi} H_{x}(x, y, z) \sin \left(\varphi+\varphi_{0}\right) d \varphi,
\end{aligned}
$$

where $\xi=\frac{q}{A} \frac{e \lambda}{2 \pi m_{e} c^{2}(\beta \gamma)_{0}}, \chi=\mu_{0} c \beta_{0} \xi=4 \pi \times 10^{-7} \times$ $c \beta_{0} \xi, m_{e}$ is the atomic unit mass, $\gamma$ is the relativistic factor, $\lambda$ is the wavelength of the rf field, and $\mu_{0}$ is the magnetic permeability of vacuum. The subindex 0 refers to the initial particle parameters, at the beginning of each integration step. The right-hand side of the equations are functions of the phase angle $\psi$. The six particle coordinates are $\left\{x, x^{\prime}, y, y^{\prime}, \varphi, \beta\right\}$, where $x, y(\mathrm{~cm})$ are particle coordinates in physical space, $x^{\prime}=\frac{p_{x}}{p}, y^{\prime}=\frac{p_{y}}{p}(\mathrm{rad})$ are the particle trajectory angles with respect to the longitudinal axis, and $\varphi_{0}(\mathrm{rad})$ is the initial phase of the particle with respect to the rf field. The TRACK code uses 48 mesh elements per accelerating cell of length $\beta_{G} \lambda / 2$, eight mesh elements in the radial direction, and 16 azimuthal mesh elements. $\beta_{G}$ is the geometrical beta of the cavity.

In each longitudinal step of integration, Eqs. (2) are solved iteratively assuming a linear dependence of the field components on the longitudinal coordinate $z$. The increment in particle coordinates is taken from the analytical solution of Eqs. (2) after the second iteration. In the first iteration, we assume $\psi=\frac{2 \pi \Delta z}{\beta_{0} \lambda}$, where $\Delta z$ is the longitudinal step along the accelerating cell. The code includes ray tracing of particles through standard optical elements such as quadrupole magnets and solenoids, bending magnets, etc.

\section{BEAM SIMULATION IN SC LINAC BASED ON QUARTER-WAVE CAVITIES}

The TRACK code was used to simulate a section of the RIA driver linac containing forty-two $115 \mathrm{MHz}$ cavities. The focusing lattice is comprised of groups of three QWRs followed by an $18 \mathrm{~cm}$ long SC solenoid. The longitudinal emittance in the driver linac is determined by a multiharmonic buncher and a $57.5 \mathrm{MHz}$ RFQ and it is as small as $2 \pi \mathrm{keV} / \mathrm{u}$ nsec [6]. Uranium beam acceleration from $4.6 \mathrm{MeV} / \mathrm{u}$ to $9.3 \mathrm{MeV} / \mathrm{u}$ for charge states 28 and 29 was simulated through 42 QWRs. To conservatively estimate the effects of steering, the input longitudinal emittance was made intentionally larger than the expected value by setting it equal to $4 \pi \mathrm{keV} / \mathrm{u}$ nsec. The results of these simulations are shown in Fig. 3. The transverse emittance growth due to the dipole components of the electromagnetic field was less than $10 \%$. However, there is some beam steering which is clearly seen in the transverse phase space plots of the output $9.3 \mathrm{MeV} / \mathrm{u}$ uranium beam shown in Fig. 3. 

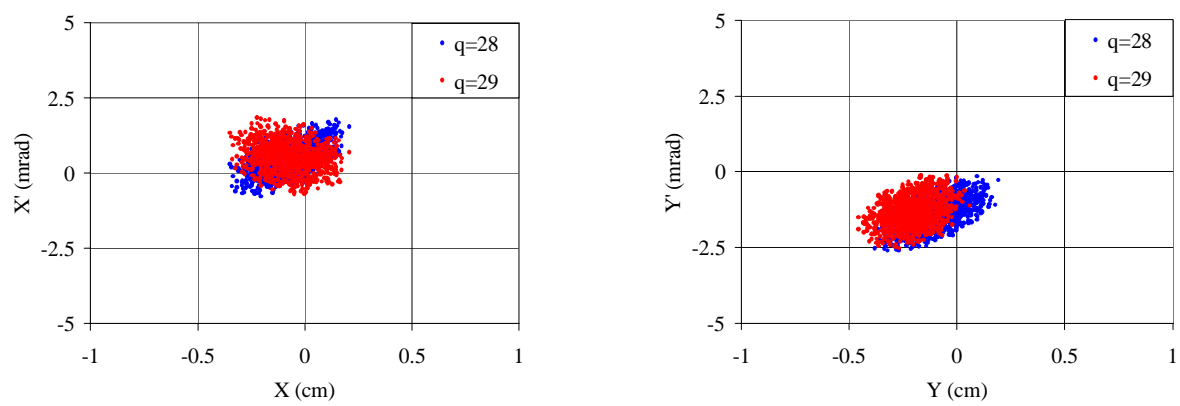

FIG. 3. (Color) Transverse phase space plots of uranium beam at charge states 28 and 29 simulated through 42 QWRs.

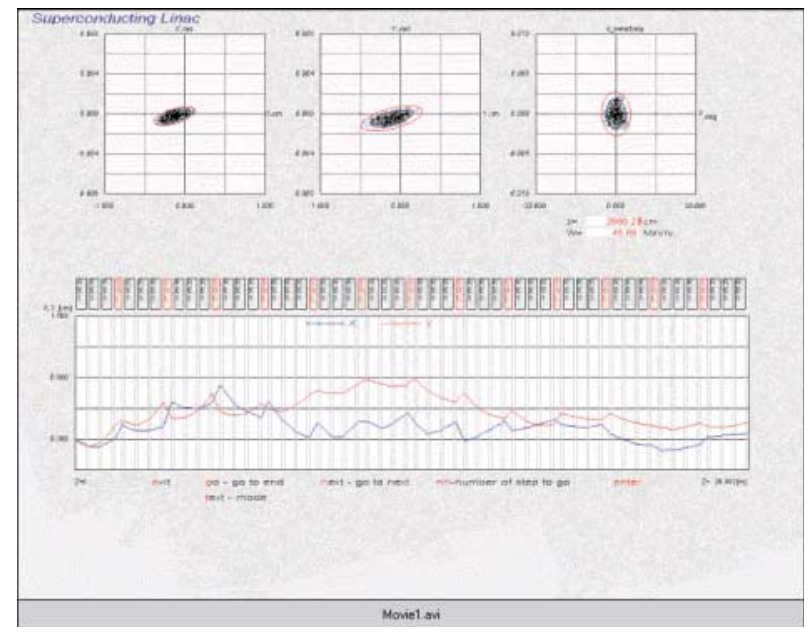

FIG. 4. (Video) Proton beam motion in the driver linac section containing $115 \mathrm{MHz}$ QWRs.

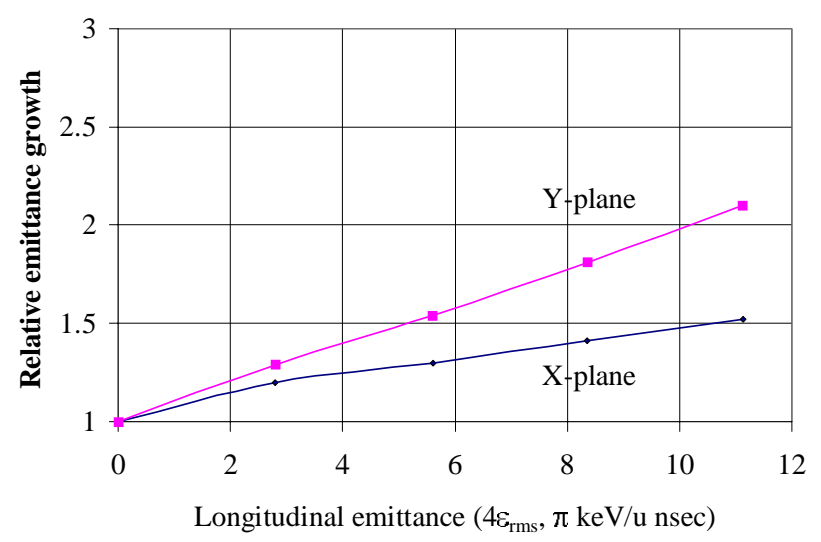

FIG. 5. (Color) Transverse rms emittance growth of a proton beam accelerated through 42 QWRs as a function of longitudinal emittance.

The compensation of the steering effect becomes particularly important if one uses SRF cavities for accelerating light ions. In this case, steering effects become more serious. Magnetic steering is enhanced because of higher specific charge and higher velocities. In a SC linac with independently phased cavities, retuning the cavity phases enables lighter, higher specific-charge ions to be accelerated to higher energy per nucleon. For example, protons can be accelerated up to $45.8 \mathrm{MeV} / \mathrm{u}$ in the section of $115 \mathrm{MHz}$ accelerating structures being considered.

Figure 4 presents an animation of the $z$ dependence of the proton beam motion along the linac. As can be seen, the steering becomes critical very quickly after injection and must be corrected in order to avoid beam loss. The solenoid focusing elements couple transverse horizontal and vertical motion, resulting in beam deflection in the horizontal plane. In addition to beam deflection, transverse emittance growth of the proton beam was studied as a function of longitudinal emittance in the multiparticle simulations. As shown in Fig. 5, we find appreciable transverse emittance growth for a proton beam which is proportional to the longitudinal emittance.

\section{ANALYSIS OF THE STEERING EFFECT}

For the understanding of the beam-steering effect and analysis of possible methods of compensation it is useful to simplify Eqs. (2). Neglecting the change in particle velocity through the cavity, a beam deflection angle in the vertical plane $\alpha=y^{\prime}$ can be determined from the equation

$$
\begin{aligned}
\frac{d \alpha}{d z}=C_{0} & {\left[E_{y}(y, z) \cos \left(k z+\varphi_{0}\right)\right.} \\
& \left.+\beta c \mu_{0} H_{x}(z) \sin \left(k z+\varphi_{0}\right)\right],
\end{aligned}
$$

where $C_{0}=\frac{q e}{A m_{e} c^{2} \beta^{2} \gamma}, k=2 \pi / \beta \lambda$. The magnetic field in the cavity is delayed by $90^{\circ}$ with respect to the electric one; therefore it is represented by a sinusoidal time dependent function. The vertical component of the electric field near the axis can be presented as a superposition of a dipole component $E_{y d}(z)$, which is approximately uniform within the aperture and a radial field which is a linear function of the vertical coordinate $y_{0}$ :

$$
E_{y}(y, z) \approx E_{y d}(z)-\frac{1}{2} \frac{\partial E_{z}(z)}{\partial z} y_{0} .
$$

If a particle enters the cavity off axis, the steering $\alpha_{\mathrm{TOT}}$ can be presented as three main components: (i) steering $\alpha_{E d}$ due to the vertical electric field, (ii) steering $\alpha_{E f}$ due to the defocusing (or focusing in dependence from $\mathrm{rf}$ phase) field, and (iii) magnetic steering $\alpha_{H}$ : 


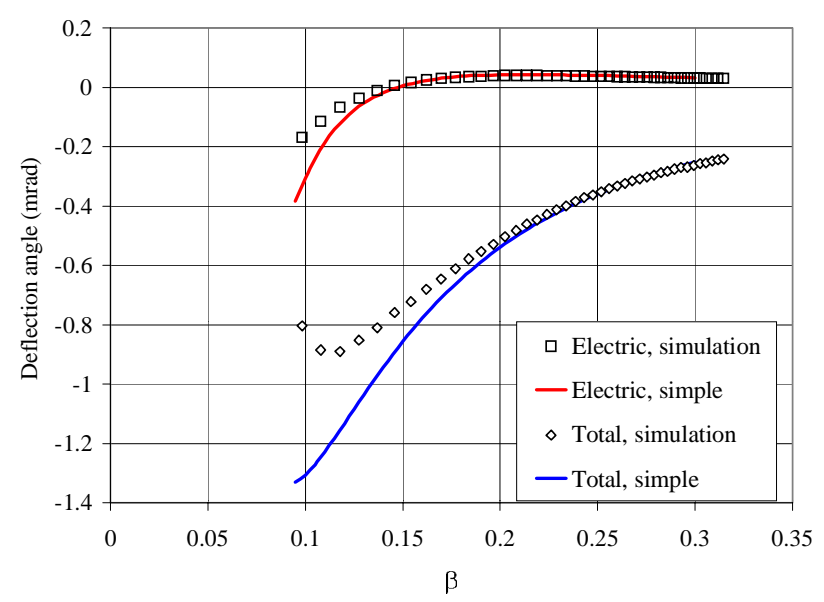

FIG. 6. (Color) Proton beam deflection angle in $115 \mathrm{MHz}$ QWR calculated by simplified Eqs. (6)-(8) and ray-tracing code TRACK. The upper curve corresponds to electric steering and the bottom curve to the total steering. The TRACK simulations are shown by discrete symbols.

$$
\begin{gathered}
\alpha_{\mathrm{TOT}}=\alpha_{E d}+\alpha_{E f}+\alpha_{H}, \\
\alpha_{E d}=-C_{0} \int_{-L / 2}^{L / 2} E_{y d}(z) \sin (k z+\varphi) d z, \\
\alpha_{E f}=\frac{y_{0} C_{0}}{2} \int_{-L / 2}^{L / 2} \frac{\partial E_{z}(z)}{\partial z} \sin (k z+\varphi) d z, \\
\alpha_{H}=\beta c \mu_{0} C_{0} \int_{-L / 2}^{L / 2} H_{x}(z) \cos (k z+\varphi) d z,
\end{gathered}
$$

where $L$ is the length of cavity; for the simplicity of further computations we assume $L=\beta_{G} \lambda$. In Eqs. (6) $-(8)$ we used $\varphi=\varphi_{0}-\frac{\pi}{2}$ for the reason that the maximum acceleration occurs at $\varphi=0$. Because our calculations are made in the standing wave approximation, the syn-

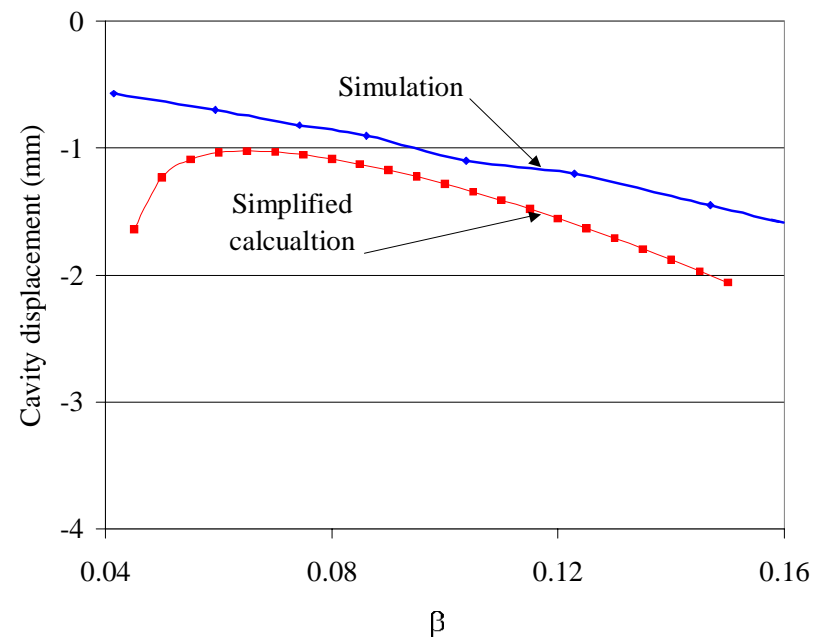

FIG. 7. (Color) A displacement of the $57.5 \mathrm{MHz}$ QWR needed for compensation of the steering. The red line corresponds to the calculations based on Eqs. (6)-(8), and the blue curve is based on the TRACK code.

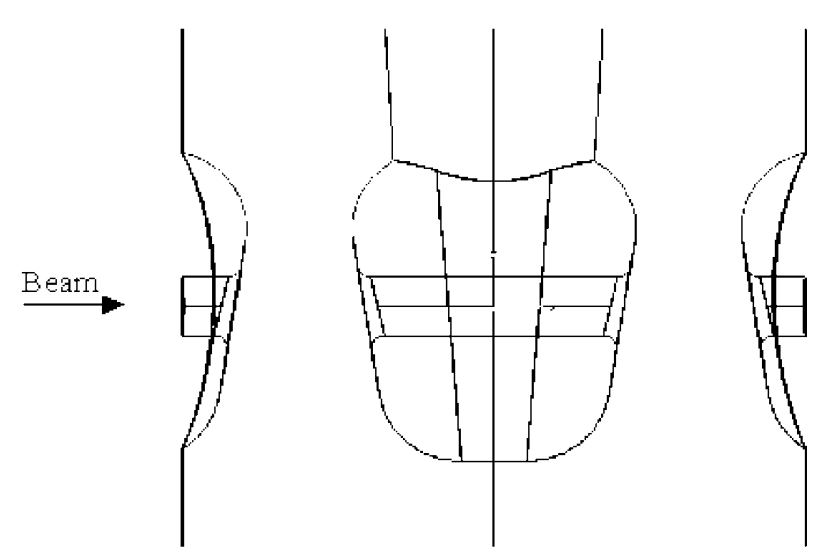

FIG. 8. $115 \mathrm{MHz}$ QWR with modified shapes of drift tubes.

chronous phase is negative. Beam steering due to the defocusing component of the electric field occurs only if the beam enters the cavity off axis with a displacement $y_{0}$.

A qualitative method of compensating the beam-steering effect in QWRs can be clearly seen if one considers the most pronounced terms of the Fourier series of the fields components $E_{y d 0}(z), E_{z 0}(z)$, and $H_{x 0}(z)$ along the $z$ axis over the period $L$. As is clear from Fig. 2, these terms of the Fourier series are

$$
\begin{aligned}
E_{y d}(z) & \approx E_{y d 0} \approx \mathrm{const}, \\
E_{z}(z) & \approx-E_{z 0} \sin k z, \\
H_{x}(z) & \approx-H_{x 0} \sin k z .
\end{aligned}
$$

Substituting Eq. (9) into Eqs. (6)-(8) one finds that all beam-steering components are in phase or $180^{\circ}$ out of phase.

Equations (6)-(8) can be used for simplified analysis of the contribution of each component to the beam deflection in QWR. Figure 6 shows the dependence of these components on the relative velocity of the proton beam

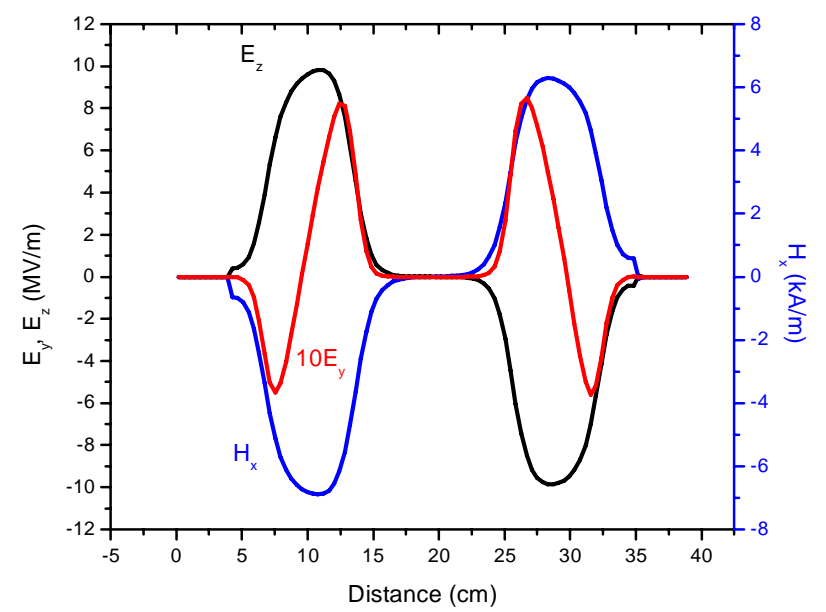

FIG. 9. (Color) Field distribution along the axis of the $115 \mathrm{MHz}$ QWR modified to correct beam steering. For clarity, the magnetic field is shown with opposite sign with respect to the electric field. The amplitude of $E_{y}$ is multiplied by a factor of 10 . 

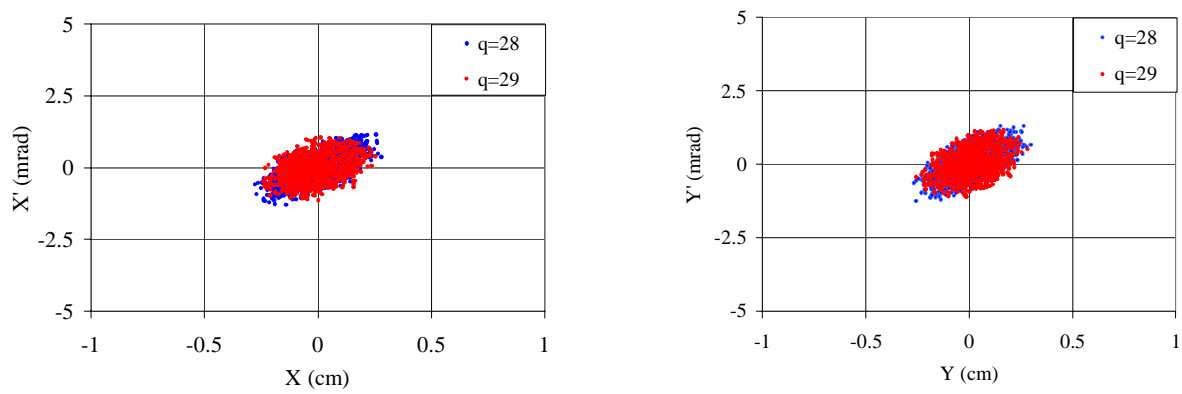

FIG. 10. (Color) Transverse phase space plots of the uranium beam with charge states 28 and 29 simulated through 42 QWRs modified as shown in Fig. 8.

being accelerated in $115 \mathrm{MHz}$ QWRs at $-20^{\circ}$ of synchronous phase. Comparison of simplified calculations (dotted curves in Fig. 6) with the simulation of the exact equation of motion by TRACK shows that the expressions (6) $-(8)$ work well above the geometrical beta $\beta_{G}$ of the cavity, which is equal to 0.15 in this particular case.

One method to compensate beam steering in a QWR is to displace the cavity in the vertical direction. Namely, the aperture center of a QWR must be displaced down with respect to the general accelerator axis. Taking into account (9) and comparing Eqs. (7) and (8) we can see that the magnetic dipole steering can be completely compensated both in phase and amplitude by an initial vertical offset of the cavities with respect to the beam axis. The value of the cavity offset can be found from (7)-(9) or more precisely by the TRACK simulations. The steering by the cavity displacement can correct both the electric and magnetic steering. Figure 7 shows the cavity displacement needed for compensation of the steering effect in $57.5 \mathrm{MHz}$ QWRs with $\beta_{G}=0.06$. As can be seen, a total compensation of the steering effect is achieved by the cavity displacement less than $2 \mathrm{~mm}$ over a wide range of beam velocity.

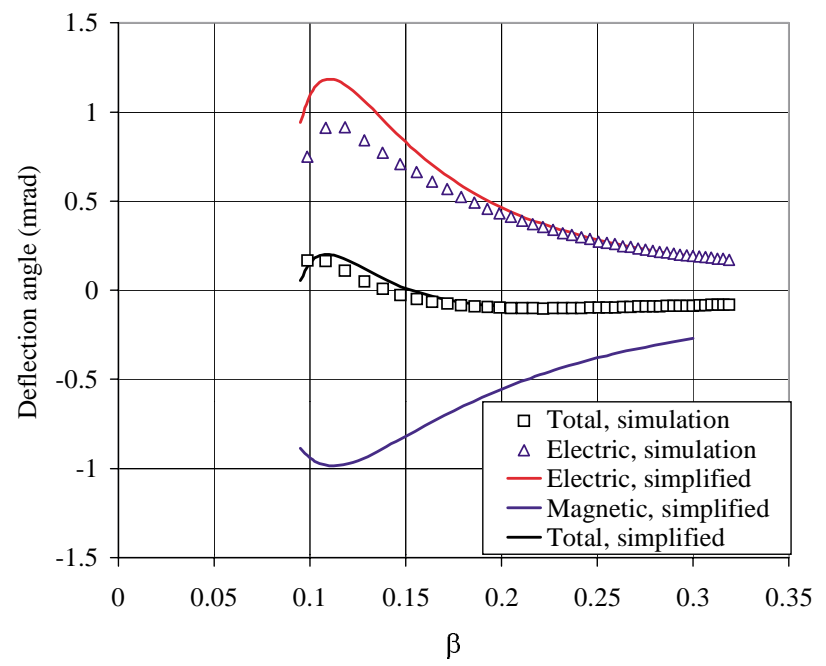

FIG. 11. (Color) Proton beam deflection angle in $115 \mathrm{MHz}$ QWRs calculated by the simplified equations (6)-(8) (solid curves) and ray-tracing code TRACK (dotted curves).
This complete compensation of the steering effect can be done for the given velocity profile. If the cavities are tuned for different ion species $q / A$ it will result in different velocity profiles so that the complete compensation cannot be achieved by this method. The steering compensation by cavity displacement can be applied effectively for low beam velocities.

Another method of compensation of the beam steering is to introduce a dipole component of vertical electric field which adds a strong $\cos (n k z)(n=0,1, \ldots)$ term into the Fourier series of equations (9). Such a distribution can be obtained by tilting the drift tube faces as shown in Fig. 8. The corresponding field distributions are shown in Fig. 9. The correct tilt angle of the drift tube faces was found by an iterate procedure. The particle motion was analyzed initially by the simplified expressions and finally by the ray-tracing code using the field distributions obtained by MWS.

Multiparticle simulation of uranium and proton beams was carried out in the linac with modified, steeringcorrected QWRs. Figure 10 shows the transverse phase space plots of a two-charge-state uranium beam at the exit of this section of the linac. Comparison with Fig. 3

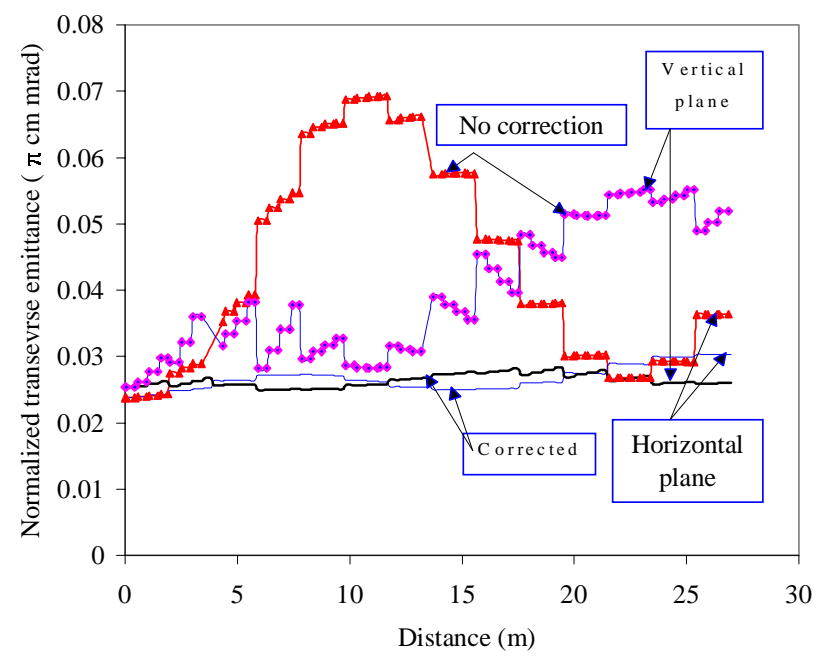

FIG. 12. (Color) Transverse emittance growth in the section of driver linac containing 42 cavities of $115 \mathrm{MHz}$ QWR with and without the compensation of beam-steering effect. 
indicates that the uranium beam steering is completely corrected.

The results of proton beam simulations are shown in Figs. 11 and 12. The final shape of the drift tubes does not completely eliminate, but does greatly reduce, the steering, as seen in Fig. 11. Transverse emittance growth, however, is almost completely eliminated, as shown in Fig. 12. The residual steering of the beam is a weak function of particle velocity as seen from Fig. 11, which means there is small dependence from charge-to-mass ratio.

\section{CONCLUSIONS}

Steering in QWR cavities can cause not only beam deflection, but also a level of transverse emittance growth which is unacceptable for many applications. We found that the steering can be largely compensated by either of two different methods. Simply offsetting the cavities by a few $\mathrm{mm}$ can often provide adequate compensation. In this method an available range of steering is limited by the useful aperture of the linac. This method does not require a manufacture of new types of cavities and can be applied for heavy-ion accelerators dealing with $\frac{q}{A} \leq \frac{1}{3}$ in velocity range $\sim 0.01 c-0.15 c$. More generally, steering can be largely eliminated by shaping the drift-tube and cavity-wall faces adjacent to the beam axis to provide appropriate corrective vertical electric field components. This method is more universal and can correct steering of pro- ton beams over the entire velocity range where QWRs are applicable.

\section{ACKNOWLEDGMENTS}

The authors thank A.A. Kolomiets for helpful assistance during the development of the beam dynamics simulation code. This work is supported by the U.S. Department of Energy, Nuclear Physics Division, under Contract No. W-31-109-ENG-38.

[1] K. W. Shepard et al., in Proceedings of the 9th Workshop on RF Superconductivity, Santa Fe, NM, 1999, edited by B. Rusnak (LANL, Los Alamos, 1999), Vol. 2, p. 345.

[2] CST Microwave Studio, User Manual Version 3.0, CST GmbH, Darmstadt, Germany, 2001, http://www.cst.de

[3] A. Facco and V. Zviagintsev, in Proceedings of the 2001 Particle Accelerator Conference, Chicago, IL (to be published); http://pacwebserver.fnal.gov/papers/Monday/ PM_Poster/MPPH136.pdf

[4] D. V. Gorelov and P. N. Ostroumov, in Proceedings of the Fifth European Particle Accelerator Conference, Sitges Spain, edited by R. Pascual (Universitat Autonoma de Barcelona, Barcelona, 1996), p. 1271.

[5] D. V. Gorelov, TRIUMF Design Note No. TRI-DN-97-06, 1997.

[6] P. N. Ostroumov et al., in Proceedings of the 2000 Linac Conference, Monterey, CA, edited by A. W. Chao (SLAC, Stanford, 2000), p. 202. 